\title{
Geostatistical inversion of seismic and ground-penetrating radar reflection images: What can we actually resolve?
}

\author{
James Irving $^{1}$ and Klaus Holliger ${ }^{2}$ \\ Received 26 July 2010; revised 7 September 2010; accepted 15 September 2010; published 6 November 2010.
}

[1] Estimation of the spatial statistics of subsurface velocity heterogeneity from surface-based geophysical reflection survey data is a problem of significant interest in seismic and ground-penetrating radar (GPR) research. A method to effectively address this problem has been recently presented, but our knowledge regarding the resolution of the estimated parameters is still inadequate. Here we examine this issue using an analytical approach that is based on the realistic assumption that the subsurface velocity structure can be characterized as a band-limited scale-invariant medium. Our work importantly confirms recent numerical findings that the inversion of seismic or GPR reflection data for the geostatistical properties of the probed subsurface region is sensitive to the aspect ratio of the velocity heterogeneity and to the decay of its power spectrum, but not to the individual values of the horizontal and vertical correlation lengths. Citation: Irving, J., and $\mathrm{K}$. Holliger (2010), Geostatistical inversion of seismic and groundpenetrating radar reflection images: What can we actually resolve?, Geophys. Res. Lett., 37, L21306, doi:10.1029/2010GL044852.

\section{Introduction}

[2] It is generally more suitable to characterize strongly heterogeneous subsurface environments using a stochastic, rather than deterministic, approach. This means that we estimate parameters describing the geostatistical nature of the heterogeneity, rather than a detailed distribution of material properties. To this end, estimation of the geostatistical properties of subsurface velocity heterogeneity from surface-based seismic and ground-penetrating radar (GPR) reflection images has been a long-standing problem of significant interest [e.g., Holliger et al., 1992; Hurich, 1996; Pullammanappallil et al., 1997; Rea and Knight, 1998; Bean et al., 1999; Poppeliers and Levander, 2004; Carpentier and Roy-Chowdhury, 2007; Knight et al., 2007]. Of particular interest has been the estimation of the lateral statistics of a subsurface velocity field from those of the corresponding reflection image, as this information cannot be obtained from borehole log or core analysis. A primary motivation for this work has been the prospect of having an effective means of obtaining realistic geostatistical models of inaccessible subsurface regions, which could greatly facilitate the understanding, characterization, and modeling of such diverse environments as the crystalline crust, hydro-

\footnotetext{
${ }^{1}$ School of Engineering, University of Guelph, Ontario, Canada.

${ }^{2}$ Institute of Geophysics, University of Lausanne, Lausanne, Switzerland.
}

Copyright 2010 by the American Geophysical Union. 0094-8276/10/2010GL044852 carbon reservoirs, groundwater aquifers, the vadose zone, and mining prospects.

[3] Previous work on the problem of estimating subsurface geostatistical properties from seismic or GPR reflection data has shown that the second-order spatial statistics of the data are indeed related to those of the underlying heterogeneous subsurface velocity distribution. However, such work has either been largely empirical or methodologically inadequate, and a rigorous means of linking the statistical properties of depth-imaged reflection data to the geostatistical properties of velocity has only recently been presented. Irving et al. [2009, 2010] describe the corresponding methodology and provide a comprehensive review of previous work on this topic. With the availability of this method and the development of an effective inversion approach, however, have come some surprising and somewhat enigmatic observations. Most notably, there is evidence to suggest that the second-order spatial statistics of seismic or GPR reflection images are sensitive only to the structural aspect ratio of the velocity heterogeneity, and not to the horizontal and vertical correlation lengths individually. Consequently, it may not be possible to resolve separately the horizontal and vertical correlation lengths of the underlying velocity structure from a reflection image. Another important, and as of yet unresolved, question concerns the sensitivity with regard to the decay of the power spectrum characterizing the fine-scale details of the velocity heterogeneity.

[4] In this paper, we explore these questions using an analytical approach that is based on the realistic assumption that the subsurface velocity heterogeneity obeys some generic band-limited scaling laws and hence can be characterized by a van-Karman-type power spectrum. Our goal is to concretely demonstrate just what, and what not, we may ideally hope to recover regarding the stochastic properties of velocity from those of the corresponding seismic or GPR reflection image. We first review the methodological background for our approach, and then analytically assess the sensitivity of the 2-D power spectrum of a geophysical reflection image to the parameters describing the underlying von-Karman-type heterogeneity. We then test and verify our analytical work with a series of numerical examples.

\section{Methodological Background}

[5] In the absence of multiple scattering, the ideal seismic or GPR reflection depth-image, $d$, commonly referred to as the primary reflectivity section (PRS), can be described as the convolution of the subsurface reflection coefficient distribution, $r$, with a source wavelet, $w$ [e.g., Claerbout, 1985]. This model can be further generalized to account for the lateral resolution limitations of a depth-migrated 
image through convolution with a corresponding horizontal resolution filter, $h$ [e.g., Scholer et al., 2010]. In the depth domain, this is expressed as follows:

$$
\begin{aligned}
d(x, z) & \approx \begin{array}{c}
r(x, z) * w(z) * h(x) \\
\end{array} \\
& \approx \frac{\partial \Delta v(x, z)}{\partial z} * w(z) * h(x)
\end{aligned}
$$

where $*$ is the convolution operator and $\Delta v$ denotes the small-scale stochastic velocity variations around the largescale deterministic trend. Equation (1) leads us to the following relationships between the corresponding 2-D power spectra, $P$ :

$$
\begin{aligned}
& P_{d}\left(k_{x}, k_{z}\right)=P_{r}\left(k_{x}, k_{z}\right) \cdot P_{w}\left(k_{z}\right) \cdot P_{h}\left(k_{x}\right) \\
& =k_{z}^{2} P_{v}\left(k_{x}, k_{z}\right) \cdot P_{w}\left(k_{z}\right) \cdot P_{h}\left(k_{x}\right),
\end{aligned}
$$

where the subscripts $d, r, w, h$, and $v$ denote the data, reflectivity series, wavelet, horizontal resolution filter, and velocity, respectively, and $k_{x}$ and $k_{z}$ are the horizontal and vertical wavenumbers. Note that, despite its conceptual simplicity, the PRS model given by equations (1) and (2) has been found to provide remarkably accurate and realistic approximations of the images obtained by depthmigrating full-waveform simulations of seismic or GPR reflection data [e.g., Irving et al., 2009, 2010; Scholer et al., 2010].

[6] There is increasing evidence to suggest that smallscale seismic and GPR velocity variations in the earth are governed by ubiquitous and seemingly universal bandlimited scaling laws [e.g., Holliger, 1996; Ulrych, 1999], which can be described through a generic von-Karman-type power spectrum of the form

$$
P_{v}\left(k_{x}, k_{z}\right)=\frac{c}{\left(k_{x}^{2} a_{x}^{2}+k_{z}^{2} a_{z}^{2}+1\right)^{(\gamma+1)}},
$$

where $c$ is a constant, $a_{x}$ and $a_{z}$ are the horizontal and vertical correlation lengths, respectively, and $0 \leq \gamma \leq 1$ controls the nature of the scaling properties. This family of power spectra is white for $k_{i}^{2} a_{i}^{2} \ll 1$ and exhibits a power-law decay typical of scale-invariant, or fractal, media for $k_{i}^{2} a_{i}^{2} \gg 1$. It is interesting to note that for $\gamma=0.5$, the von-Karman power spectrum is equivalent to the widely used exponential variogram model of geostatistics. Also note that this heterogeneity model and our considerations based on it can be readily generalized to the case where the structural grain is not aligned with the considered Cartesian coordinate system.

[7] Substituting equation (3) into equation (2) and making use of the relationship $\alpha=a_{x} / a_{z}$, where $\alpha$ denotes the structural aspect ratio of the velocity heterogeneity, we obtain the following equation for the power spectrum of a seismic or GPR reflection depth-image:

$$
P_{d}\left(k_{x}, k_{z}\right)=\frac{c k_{z}^{2}}{\left(k_{x}^{2} \alpha^{2} a_{z}^{2}+k_{z}^{2} a_{z}^{2}+1\right)^{(\gamma+1)}} \cdot P_{w}\left(k_{z}\right) \cdot P_{h}\left(k_{x}\right) .
$$

Let us now consider the case where, in the vertical dimension, the band-limitation provided by the seismic or GPR source wavelet places us in the regime where scale-invariance prevails. In other words, let us assume that the source wavelet restricts us to a region of the vertical power spectrum where $k_{z}^{2} a_{z}^{2} \gg 1$. This is roughly equivalent to saying that the dominant wavelength is much smaller than the vertical correlation scale of the subsurface velocity field, and thus primarily senses its smaller-scale, fractal-type heterogeneity. This is indeed a realistic assumption upon which most standard seismic and GPR deconvolution and imaging algorithms are based [e.g., Yilmaz, 1987; Ulrych, 1999], and leads to the following simplification of equation (4):

$$
P_{d}\left(k_{x}, k_{z}\right) \approx \frac{c k_{z}^{2}}{a_{z}^{2(\nu+1)}\left(\alpha^{2} k_{x}^{2}+k_{z}^{2}\right)^{(\gamma+1)}} \cdot P_{w}\left(k_{z}\right) \cdot P_{h}\left(k_{x}\right)
$$

[8] The above expression can now be used to examine the sensitivity of the power spectrum of imaged seismic or GPR reflection data to the various parameters describing the underlying velocity heterogeneity.

\section{Sensitivity Analysis}

[9] To explore the sensitivity of $P_{d}\left(k_{x}, k_{z}\right)$ in equation (5) to the parameters describing the stochastic velocity heterogeneity, $a_{z}, \alpha$, and $\gamma$, we consider the following spectral ratio:

$$
\frac{P_{d 2}\left(k_{x}, k_{z}\right)}{P_{d 1}\left(k_{x}, k_{z}\right)}=\frac{a_{z 1}^{2\left(\gamma_{1}+1\right)}\left(\alpha_{1}^{2} k_{x}^{2}+k_{z}^{2}\right)^{\left(\gamma_{1}+1\right)}}{a_{z 2}^{2\left(\gamma_{2}+1\right)}\left(\alpha_{2}^{2} k_{x}^{2}+k_{z}^{2}\right)^{\left(\gamma_{2}+1\right)}},
$$

where the subscripts 1 and 2 represent before and after a given parametric change, respectively. The first case we wish to examine involves different vertical correlation lengths but constant aspect ratios and $\gamma$-values, as described by

$$
a_{z 1} \neq a_{z 2}, \alpha_{1}=\alpha_{2}=\alpha, \gamma_{1}=\gamma_{2}=\gamma .
$$

It is important to note that a change in the vertical correlation length with no change in the aspect ratio implies a corresponding change in the horizontal correlation length in equation (3). As a result, in this case we are testing how the power spectrum of a reflection image is affected by changes in both the vertical and horizontal correlation lengths such that the aspect ratio remains constant. Under these conditions, the spectral ratio in equation (6) simplifies to

$$
\frac{P_{d 2}\left(k_{x}, k_{z}\right)}{P_{d 1}\left(k_{x}, k_{z}\right)}=\left(\frac{a_{z 1}}{a_{z 2}}\right)^{2(\gamma+1)}=\text { constant. }
$$

Equation (7) is a key result, as it importantly confirms the recent empirical findings of Irving et al. [2009, 2010] and Scholer et al. [2010] suggesting that seismic or GPR images derived from velocity structures having the same aspect ratio, but different correlation lengths, will show no change in the shape of their 2-D power spectra, or equivalently their 2-D autocorrelations or variograms. Consequently, the corresponding inverse problem is non-unique and it will not be possible to estimate the individual horizontal and vertical correlation lengths of the velocity heterogeneity from the second-order statistics of a seismic or GPR reflection image, as has been previously investigated.

[10] The next case we wish to examine involves different aspect ratios, or equivalently different horizontal correlation 


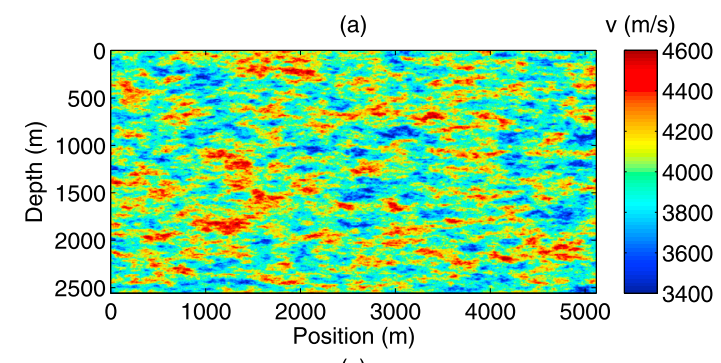

(c)

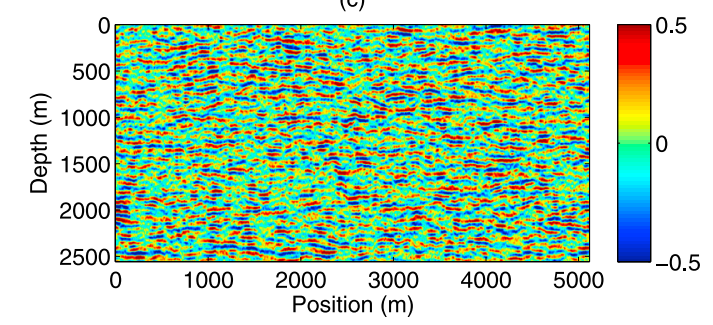

(b)

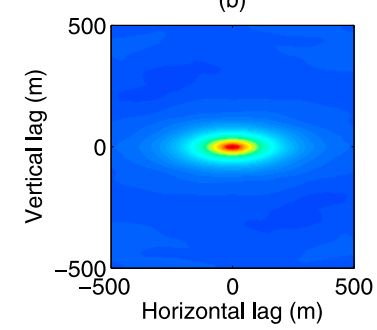

(d)

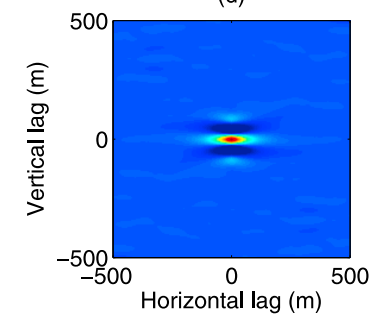

Figure 1. (a) Seismic velocity model characterized by $a_{x}=120 \mathrm{~m}, a_{z}=40 \mathrm{~m}, \gamma=0.5$. (b) 2-D autocorrelation of seismic velocity distribution. (c) Ideal $20-\mathrm{Hz}$ seismic depth-image given by PRS. (d) 2-D autocorrelation of PRS.

lengths, in conjunction with constant $\gamma$-values and vertical correlation lengths, as described by

$$
\alpha_{1} \neq \alpha_{2}, \gamma_{1}=\gamma_{2}=\gamma, a_{z 1}=a_{z 2}=a_{z}
$$

This yields for the ratio of the power spectra of the imaged seismic or GPR reflection data:

$$
\frac{P_{d 2}\left(k_{x}, k_{z}\right)}{P_{d 1}\left(k_{x}, k_{z}\right)}=\left(\frac{\alpha_{1}^{2} k_{x}^{2}+k_{z}^{2}}{\alpha_{2}^{2} k_{x}^{2}+k_{z}^{2}}\right)^{(\gamma+1)}
$$

Here we see that changing the aspect ratio of the velocity heterogeneity results in a pronounced change in the power spectrum, and thus of the autocorrelation function, of the corresponding seismic or GPR reflection image. This in turn shows that it should be possible to recover information about the aspect ratio of the velocity heterogeneity from the second-order statistics of the image, which is also consistent with recent empirical findings.

[11] The third and last case we wish to consider involves different $\gamma$-values in combination with constant aspect ratios and vertical correlation lengths, as described by

$$
\gamma_{1} \neq \gamma_{2}, \alpha_{1}=\alpha_{2}=\alpha, a_{z 1}=a_{z 2}=a_{z} .
$$

This results in the following simplification of the spectral ratio in equation (6):

$$
\frac{P_{d 2}\left(k_{x}, k_{z}\right)}{P_{d 1}\left(k_{x}, k_{z}\right)}=a_{z}^{2\left(\gamma_{1}-\gamma_{2}\right)}\left(\alpha^{2} k_{x}^{2}+k_{z}^{2}\right)^{\left(\gamma_{1}-\gamma_{2}\right)},
$$

which indicates that the power spectrum or autocorrelation of a reflection image should also be sensitive to changes in

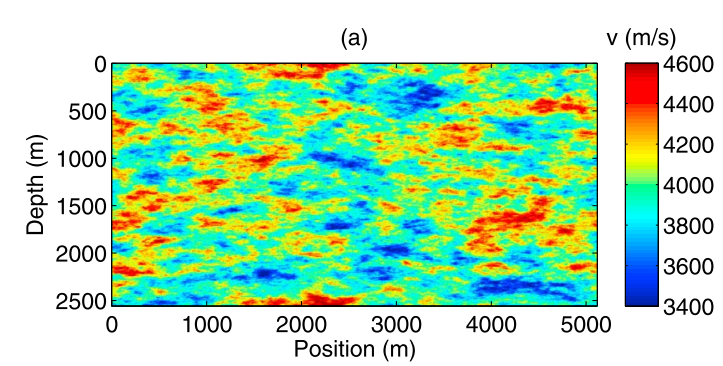

(c)

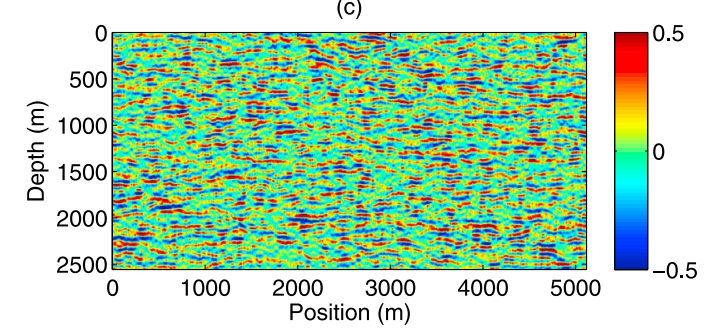

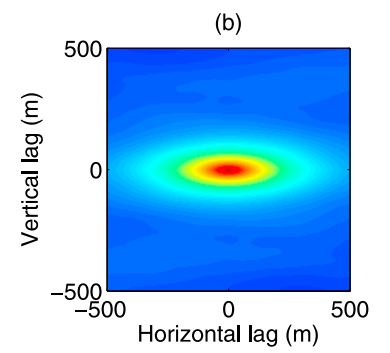

(d)

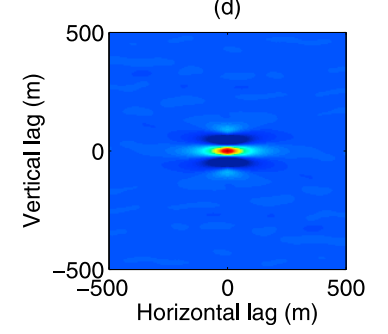

Figure 2. (a) Seismic velocity model characterized by $a_{x}=240 \mathrm{~m}, a_{z}=80 \mathrm{~m}, \gamma=0.5$. (b) 2-D autocorrelation of seismic velocity distribution. (c) Ideal $20-\mathrm{Hz}$ seismic depth-image given by PRS. (d) 2-D autocorrelation of PRS. 

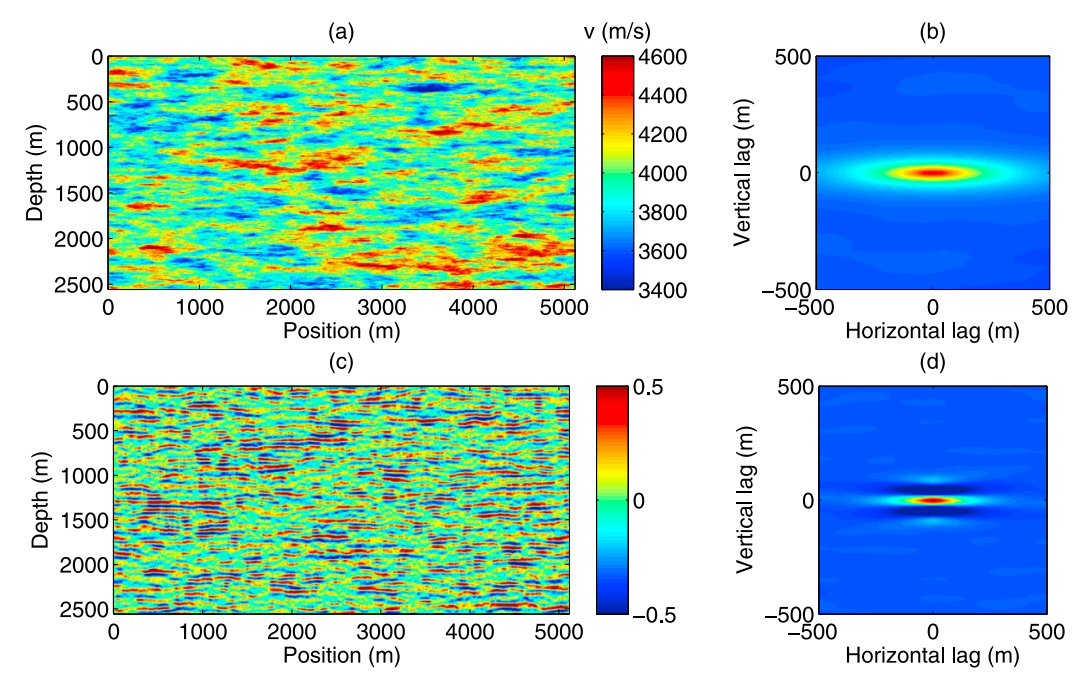

Figure 3. (a) Seismic velocity model characterized by $a_{x}=240 \mathrm{~m}, a_{z}=40 \mathrm{~m}, \gamma=0.5$. (b) 2-D autocorrelation of seismic velocity distribution. (c) Ideal $20-\mathrm{Hz}$ seismic depth-image given by PRS. (d) 2-D autocorrelation of PRS.

the $\gamma$-value describing the degree of self-similarity of the underlying velocity heterogeneity.

\section{Numerical Verification}

[12] We now seek to test and verify the results of our analytical sensitivity analysis through a series of simple yet pertinent numerical examples. In each case, we generate a stochastic velocity field for some set of values of the defining parameters $a_{x}, a_{z}$, and $\gamma$, and we calculate the corresponding PRS seismic depth-image using equation (1). We then compute and compare the corresponding 2-D autocorrelations. In this analysis, we also seek to assess the importance of our assumption that the data are dominated by the scale-invariant part of the vertical velocity structure as characterized by $k_{z}^{2} a_{z}^{2} \gg 1$. To this end, we define our reference model such that $k_{z}^{2} a_{z}^{2} \approx 1$, where $k_{z}$ is determined from the dominant frequency of the source wavelet. This implies that only approximately half of the spectral bandwidth of the wavelet will be dominated by the scaleinvariant part of the velocity structure given by equation (3), whereas the other half will be dominated by the white part of the heterogeneity spectrum. In this work, it is important to remember that the autocorrelation simply represents the inverse Fourier transform of the power spectrum. Our analytical derivations were easier to carry out in the power spectral domain because the von-Karman model in the autocorrelation domain involves a cumbersome modified Bessel function of the second kind. Conversely, the numerical results presented here are best assessed in the autocorrelation domain due its inherently limited dynamic range and corresponding ease of intuitive understanding and qualitative comparison.

[13] Figure 1 shows a subsurface velocity field characterized by band-limited scale-invariance and its corresponding PRS generated using a Ricker source wavelet with a (a)

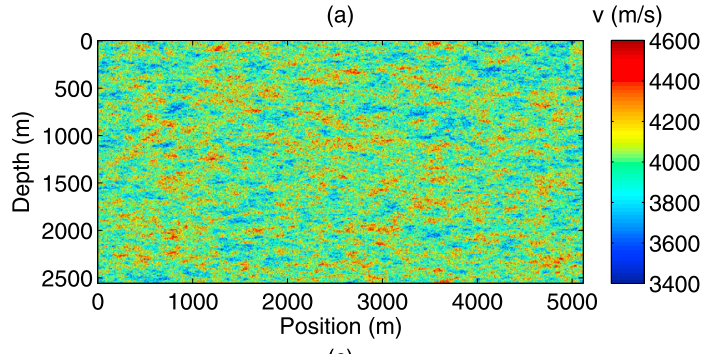

(c)

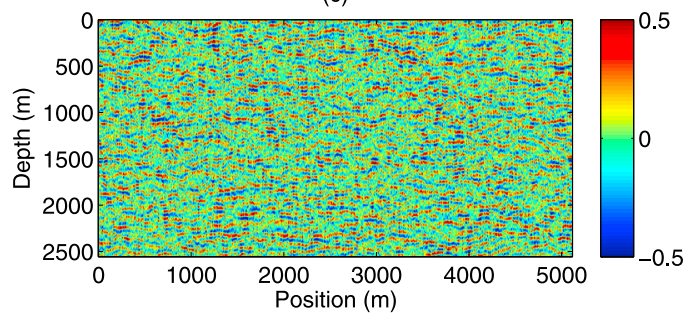

(b)

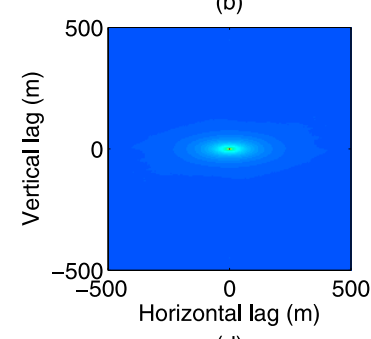

(d)

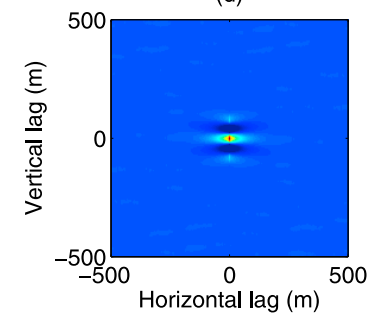

Figure 4. (a) Seismic velocity model characterized by $a_{x}=120 \mathrm{~m}, a_{z}=40 \mathrm{~m}, \gamma=0.1$. (b) 2-D autocorrelation of seismic velocity distribution. (c) Ideal 20-Hz seismic depth-image given by PRS. (d) 2-D autocorrelation of PRS. 
dominant frequency of $20 \mathrm{~Hz}$. The corresponding autocorrelations are shown to the right. The power spectrum of the seismic velocity heterogeneity is characterized by $a_{x}=120 \mathrm{~m}$, $a_{z}=40 \mathrm{~m}$, and $\gamma=0.5$. Figures $2-4$ show the same set of plots but with changes to the velocity heterogeneity parameters corresponding to the three basic cases considered in the analytical sensitivity analysis. Specifically, we first double the horizontal and vertical correlation lengths while keeping the aspect ratio and $\gamma$-value unchanged: $a_{x}=240 \mathrm{~m}, a_{z}=80$ $\mathrm{m}, \gamma=0.5$ (Figure 2). Next, we consider a change in the aspect ratio, while keeping the vertical correlation length and $\gamma$ at their reference values: $a_{x}=240 \mathrm{~m}, a_{z}=40 \mathrm{~m}, \gamma=0.5$ (Figure $3)$. Finally, we examine the effects of a change in the $\gamma$-value while keeping the other parameters unchanged: $a_{x}=120 \mathrm{~m}, a_{z}$ $=40 \mathrm{~m}$, and $\gamma=0.1$ (Figure 4).

[14] Comparing the autocorrelations in Figures 2-4 with those of the reference model in Figure 1, we see that while there are notable differences between the autocorrelations of the velocity models for all of the examples considered, this is not always the case for the autocorrelations of the corresponding PRS. In particular, we observe that the autocorrelation of the PRS in Figure 2 is nearly identical to that of the reference model in Figure 1, whereas those in Figures 3 and 4 show distinct differences. These results confirm that, even when violating the assumption of pure scale-invariance, the second-order statistics of depthimaged seismic and GPR reflection data will be sensitive to the aspect ratio of the velocity heterogeneity, but not to the individual values of the horizontal and vertical correlation lengths. Again, these findings are consistent with recent empirical work, and in fact there is strong evidence in this work to suggest that the results maintain their overall validity even in the presence of elastic scattering, standard seismic processing involving common-mid-point stacking [Irving et al., 2010], and in subsurface environments characterized by non-Gaussian and modal velocity distributions [Scholer et al., 2010]. Our numerical tests also confirm the analytical finding that the second-order statistics of a seismic or GPR reflection image should be sensitive to the power spectral slope of the velocity heterogeneity. However, the available empirical evidence regarding this result is still too thin and inconsistent to either corroborate or challenge it [e.g., Carpentier and Roy-Chowdhury, 2007; Scholer et al., 2010]. We therefore see this as an important topic for future research.

\section{Conclusions}

[15] A number of previous studies in both seismic and GPR research have investigated the question of whether the second-order statistical structure of a reflection depth-image can be used to provide information regarding the stochastic properties of the underlying velocity model. To address this issue, we have examined the parametric sensitivity of the geostatistical inversion of depth-imaged reflection data using an analytical approach followed by numerical verification. In doing so, we assumed that the subsurface velocity distribution can be characterized as a band-limited scaleinvariant medium. For our analytical considerations, we enforced strict scale-invariance in the vertical dimension, but then relaxed this condition for the numerical part of our study. Despite these differences, the analytical and numerical results are fully consistent and in agreement with recent empirical evidence. They indicate that, in the absence of any prior information, the inversion of seismic and GPR depthimages will be sensitive to the structural aspect ratio and the power spectral decay of the velocity heterogeneity, but not to the individual values of the horizontal and vertical correlation lengths. In this context, however, it is important to note that the vertical correlation length can often be constrained based on information from borehole logs, which then, via the sensitivity to the aspect ratio, allows for estimating the horizontal correlation length.

[16] Acknowledgments. This research has been supported by the Swiss National Science Foundation.

\section{References}

Bean, C. J., D. Marsan, and F. Martini (1999), Statistical measures of crustal heterogeneity from reflection seismic data: the role of seismic bandwidth, Geophys. Res. Lett., 26(21), 3241-3244, doi:10.1029/ 1999GL005400.

Carpentier, S., and K. Roy-Chowdhury (2007), Underestimation of scale lengths in stochastic fields and their seismic response: a quantification exercise, Geophys. J. Int., 169(2), 547-562, doi:10.1111/j.1365246X.2007.03333.x.

Claerbout, J. (1985), Imaging the Earth's Interior, Blackwell Sci., Boston.

Holliger, K. (1996), Upper-crustal seismic velocity heterogeneity as derived from a variety of P-wave sonic logs, Geophys. J. Int., 125(3), 813-829, doi:10.1111/j.1365-246X.1996.tb06025.x.

Holliger, K., R. Carbonell, and A. Levander (1992), Sensitivity of the lateral correlation function in deep seismic reflection data, Geophys. Res. Lett., 19(22), 2263-2266, doi:10.1029/92GL02615.

Hurich, C. (1996), Statistical description of seismic reflection wavefields: A step towards quantitative interpretation of deep seismic reflection profiles, Geophys. J. Int., 125(3), 719-728, doi:10.1111/j.1365-246X.1996. tb06019.x.

Irving, J., R. Knight, and K. Holliger (2009), Estimation of the lateral correlation structure of subsurface water content from surface-based groundpenetrating radar reflection images, Water Resour. Res., 45(12), W12404, doi:10.1029/2008WR007471.

Irving, J., M. Scholer, and K. Holliger (2010), Inversion for the stochastic structure of subsurface velocity heterogeneity from surface-based geophysical reflection images, in Advances in Near-Surface Seismology and Ground-Penetrating Radar, edited by R. Miller, J. Bradford, and K. Holliger, Soc. Explor. Geophys., Tulsa, Oklahoma, in press.

Knight, R., J. Irving, P. Tercier, G. Freeman, C. Murray, and M. Rockhold (2007), A comparison of the use of radar images and neutron probe data to determine the horizontal correlation length of water content, $A G U$ Geophys. Monogr. Ser., 171, 31-44.

Poppeliers, C., and A. Levander (2004), Estimation of vertical stochastic scale parameters in the Earth's crystalline crust from seismic reflection data, Geophys. Res. Lett., 31, L13607, doi:10.1029/2004GL019538.

Pullammanappallil, S., A. Levander, and S. Larkin (1997), Estimation of crustal stochastic parameters from seismic exploration data, J. Geophys. Res., 102(B7), 15,269-15,286, doi:10.1029/97JB01144.

Rea, J., and R. Knight (1998), Geostatistical analysis of ground-penetrating radar data: a means of describing spatial variation in the subsurface, Water Resour. Res., 34, 329-340, doi:10.1029/97WR03070.

Scholer, M., J. Irving, and K. Holliger (2010), Estimation of the correlation structure of crustal velocity heterogeneity from seismic reflection data, Geophys. J. Int., doi:10.1111/j.1365-246X.2010.04793.x, in press.

Ulrych, T. (1999), The whiteness hypothesis: Reflectivity, inversion, chaos, and Enders, Geophysics, 64, 1512-1523, doi:10.1190/1.1444655.

Yilmaz, O. (1987), Seismic Data Processing, Soc. Explor. Geophys., Tulsa, Okla.

K. Holliger, Institute of Geophysics, University of Lausanne, CH-1015, Lausanne, Switzerland. (klaus.holliger@unil.ch)

J. Irving, School of Engineering, University of Guelph, ON N1G 2W1, Canada. (irvingj@uoguelph.ca) 